\title{
Le concept du multilinguisme dans la poétique autobiographique de Vladimir Nabokov*
}

\author{
Svetlana Garziano**
}

Resumo: 0 conceito do multiliguismo, etiqueta solidamente colada à arte de Vladimir Nabokov, é muito importante para compreender sua poética autobiográfica que se desenvolve em três línguas: russo, inglês e francês. 0 artigo examina os princípios da escritura plurilíngue na obra nabokoviana através de quatro temas estruturantes: bilinguismo / multilinguismo da escritura, tradução e autotradução, relação binária das línguas russa e inglesa na autobiografia e o problema da passagem do inglês para o russo segundo o exemplo de Druguíe beregá e de Lolita. A teoria linguística de Nabokov consiste em buscar uma língua universal, a da criação artística, o sentido que se transfere de uma língua à outra pelo viés de imagens visuais. Para este escritor, a tradução deve significar e fazer aparecer na consciência do leitor aquilo que não depende de nenhum idioma: o "entre-línguas", a relação entre as línguas.
Résumé: Le concept du multilinguisme, étiquette solidement collée à l'art de Vladimir Nabokov, est très important pour comprendre sa poétique autobiographique se développant dans trois langues: russe, anglais et français. L'article examine les principes de l'écriture plurilingue dans l'œuvre nabokovienne à travers quatre thèmes structurants: bilinguisme / multilinguisme de l'écriture, traduction et autotraduction, relation binaire des langues russe et anglaise dans l'autobiographie et problème du passage de l'anglais au russe sur l'exemple de Drugie berega et de Lolita. La théorie linguistique de Nabokov consiste à rechercher une langue universelle, celle de la création artistique, le sens étant transféré d'une langue à l'autre par le biais d'images visuelles. Pour cet écrivain, la traduction doit signifier et faire apparaître à la conscience du lecteur ce qui ne relève d'aucun idiome: l'«entre-langues», le rapport entre les langues.

Palavras-chave: Vladimir Nabokov; Poética autobiográfica; Multilinguismo; Autotradução; Druguíe beregá; Lolita

Mots-clés: Vladimir Nabokov ; Poétique autobiographique ; Multilinguisme ; Auto-traduction ; Drugie berega ; Lolita 
* Artigo submetido em 16 de agosto de 2018 e aprovado em 06 de setembro de 2018.

**Svetlana Garziano, maître de conférences en langue et littérature russes à la faculté des langues, membre de l'équipe MARGE à la faculté des lettres, directrice de la Maison des langues de l'Université Jean Moulin Lyon. E-mail: svetlana.garziano@univ-lyon3.fr e concept du multilinguisme, étiquette solidement collée à l'art de Vladimir Nabokov, est très important pour comprendre sa poétique autobiographique. Un exemple en russe, tiré de Camera obscura [1932-1933], en dit long à ce sujet: «Как противно Фрида говорила об этом: «И она кричала, и она кричала», - с ужасным ударением на «и»»" ("Frieda parlait de cela d'une manière répugnante, «Et elle criait, et elle criait», en mettant un accent terrifiant sur les «i»»). Sans recours à la traduction en allemand de la réplique de la bonne, toute interprétation peut paraître étrange, car l'accent dans le verbe «кричала» tombe sur la première voyelle «a». Bien évidemment, cette phrase témoigne de l'émoi extrême du personnage Anne-Lise, mais aussi, plus généralement, du fait que les personnages de ce roman parlent allemand. L'auteur du roman traduit donc en russe la pièce de théâtre que jouent ces personnages dans sa vision mentale en allemand, tout comme Aleksandr Puškin mentionne dans Eugène Onéguine qu'il traduit la lettre de Tatiana du français en russe pour son lecteur. Cette discordance au niveau sémantique permet de percevoir l'interstice «entre-langues» dans Camera obscura, crée un pont interprétatif entre le russe et l'allemand, en intégrant ces deux langues dans un système sans langues, visuel, mental, spatialisant et immobilisant la continuité et la capacité réflexives de l'artiste.

\footnotetext{
${ }^{1}$ NABOKOV, 2001, t. 3, p. 293.

Les mots russes qui apparaissent dans cet article sont translittérés selon la norme dite «des slavistes», correspondant à la norme ISO/R9 (version 1968) de l'organisation internationale de normalisation. Sauf mention contraire, tous les textes russes cités sont traduits par l'auteur de l'article.
} 


\section{Le bilinguisme / multilinguisme de l'écriture nabokovienne}

Nous nous proposons d'entamer notre réflexion par ce que Nabokov dit au sujet d'un de ses rivaux littéraires, Joseph Conrad, et comment il se différencie par rapport à ce dernier. Dans la préface de Drugie berega ${ }^{2}$, l'autobiographe énumère les écrivains russes Avvakum, Aleksandr Puškin, Lev Tolstoj, pour faire ressortir sans aucun doute sa parenté avec la tradition autobiographique russe, tout en recourant au modèle anglais, en mentionnant le nom de Joseph Conrad:

Совершенно владея с младенчества и английским и французским, я перешел бы для нужд сочинительства с русского на иностранный язык без труда, будь я, скажем, Джозеф Конрад, который, до того, как начал писать по-английски, никакого следа в родной (польской) литературе не оставил, а на избранном языке (английском) искусно пользовался готовыми формулами. ${ }^{3}$

Possédant à la perfection depuis ma prime enfance l'anglais et le français, je serais passé sans difficulté, pour les besoins de mon écriture, du russe à une langue étrangère, si j'avais été, par exemple, Joseph Conrad, qui avant de se mettre à écrire en anglais, n'avait laissé aucune trace dans sa littérature d'origine (la littérature polonaise) et qui, dans sa langue d'adoption (l'anglais), ne fit qu'utiliser habilement des formules toutes faites. ${ }^{4}$

Quelle est la fonction de cette référence? Certes, Nabokov montre que la comparaison avec Joseph Conrad, qui est son

\footnotetext{
${ }^{2}$ L'aspect ambigu de la traduction française qui donne à la seconde version de l'autobiographie anglaise Speak, Memory [1967] le titre de l'autobiographie en russe Drugie berega, à savoir Autres rivages, nous oblige à faire quelques précisions terminologiques. Dans notre article, nous avons décidé d'utiliser le titre Drugie berega pour désigner l'autobiographie russe, le titre Autres rivages pour désigner la traduction française de la seconde version anglaise et le titre Speak, Memory pour désigner la seconde version anglaise. L'autobiographie russe n'a pas été traduite en français. L'autobiographie anglaise a été traduite en français par Yvonne Davet en 1961 et 1991.

${ }^{3}$ NABOKOV, 1978, p. 7.

${ }^{4}$ NABOKOV, 1991b, p. 393.
} 
rival sérieux sous le rapport de la langue anglaise, est sans fondement car ce dernier, selon l'autobiographe, n'aurait pas assimilé le modèle originel polonais et se serait adapté au modèle étranger anglais en recourant à des formules convenues. Cette comparaison nous aide à discerner la singularité de notre auteur qui suppose une approche active et créatrice dans la réception des modèles consistant en leur transformation et en leur fusion.

Pourtant la question de Conrad apparaît, nous semble-t-il, dans la préface russe comme une sorte de frustration que l'écrivain veut évacuer définitivement en 1954 dans son autobiographie russe. Nous motivons cette hypothèse par l'apparition de ce motif dans sa correspondance avec Edmund Wilson et dans Conclusive Evidence. La phrase «En outre, en dépit de quelques erreurs, Mr. Nabokov maîtrise l'anglais presque aussi bien que Joseph Conrad» fut ajoutée par Edmund Wilson dans son compte rendu «Nicolas Gogol» [«Nicolai Gogol», New Yorker, 9 septembre 1944] pour sa publication sous le titre de «Vladimir Nabokov sur Gogol» [«Vladimir Nabokov on Gogol»]. Dans une lettre à E. Wilson du 18 novembre 1950, Nabokov attire son attention sur cet ajout postérieur:

Ton passage sur Gogol et moi contient diverses choses (ajoutées? changées?) que je ne me souviens pas avoir vues dans la version originale. Je réfute la dernière ligne. Conrad savait manier l'anglais de tous les jours mieux que moi ; mais je connais mieux l'autre. Il ne sombre jamais jusqu'aux profondeurs de mes solécismes mais n'atteint jamais mes cimes verbales. ${ }^{5}$

Et dans une lettre du 26 mai 1951, Wilson revient sur le même sujet:

L'anglais de CE est au moins aussi bon que celui de Conrad, et il a en plus des qualités auxquelles Conrad n'aurait jamais pu prétendre. Je crois que tu as dû un peu profiter du passage au peigne fin (parfois ridiculement stupide) qu'opère le New Yorker sur la prose qu'il publie. ${ }^{6}$

\footnotetext{
${ }^{5}$ NABOKOV; WILSON, 1988, p. 280. Lettre à E. Wilson du 18 novembre 1950

${ }^{6}$ Ibidem, p. 289. Lettre de E. Wilson du 26 mai 1951.
} 
Et finalement, dans le chapitre seize de Conclusive Eviden$c e$, le critique fictif compare l'art de manier une langue étrangère littéraire de Conrad à celui de Nabokov:

Evoquer le cas de Conrad en parlant des romans de Nabokov écrits en anglais (La vraie vie de Sebastian Knight et Brisure à senestre) reviendrait à se méprendre sur la prouesse réalisée par ce dernier. Conrad - dont le style anglais était, quoi qu'on dise, une collection de clichés prétentieux n'avait pas derrière lui vingt années de participation intense à la littérature polonaise lorsqu'il se lança dans sa carrière anglaise. En revanche, lorsque Nabokov passa à l'anglais, il avait déjà écrit en russe plusieurs romans et de nombreuses nouvelles, et avait même acquis une place durable dans la littérature russe, bien que ses livres fussent interdits dans son pays natal. La seule analogie entre ces deux auteurs, c'est que tous deux auraient tout aussi bien pu opter pour le français que pour l'anglais. ${ }^{7}$

Du premier coup d'œil, l'évocation de Conrad dans la préface de Drugie berega ne s'avère être autre chose que la suite de la polémique de Nabokov engagée avec Wilson au sujet de sa comparaison avec Conrad. Mais, en examinant ce problème plus en profondeur, nous pouvons dire qu'elle traduit l'intérêt que porte notre auteur au bilinguisme, voire au multilinguisme, et, comme sa conséquence, à la traduction ou, plus exactement, à l'auto-traduction.

La singularité de l'autobiographie de Nabokov consiste justement en son multilinguisme. Elle se décline dans trois langues: russe, anglais et français, car cet auteur a écrit avec une égale virtuosité et un égal succès en ces trois langues. Dans l'interview à Jane Howard pour le Life Magazine [1964], répondant à la question du choix de la langue la plus belle, il a fait cet aveu: «Mon esprit répond: l'anglais, mon cœur: le russe, mon oreille: le français»". A la question d'Alvin Toffler «Que lisiez-vous quand vous étiez un petit garçon?» tirée de l'entretien dans Playboy [janvier 1964], Nabokov donne une réponse qui révèle la genèse de son trilinguisme acquis principalement par la lecture:

7 NABOKOV, 1999, n5 551, p. 58.

8 NABOKOV, 1985a, p. 59. 
Entre dix et quinze ans à Saint-Pétersbourg, je pense avoir lu plus de romans et de poèmes - en anglais, en russe et en français - qu'au cours d'une autre période de même durée de ma vie. [...] ... j'étais un enfant trilingue parfaitement normal vivant dans une famille avec une vaste bibliothèque. ${ }^{9}$

Cependant le romancier avoue réfléchir en images. A la question «En quelle langue pensez-vous?» posée par Peter Duval-Smith et Christopher Burstall dans l'interview donnée en juillet 1962, il répond:

Je ne pense en aucune langue. Je pense en images. Je ne crois pas que les gens pensent dans une langue particulière. Ils ne remuent pas les lèvres quand ils pensent. C'est seulement certains illettrés qui remuent les lèvres en lisant ou en ruminant. Non, je pense en images, et de temps en temps une phrase russe ou une phrase anglaise peuvent se former sur l'écume de l'onde cérébrale, mais c'est tout. ${ }^{10}$

D'après notre auteur, la pensée s'effectue en images et par elles. Pour Nabokov, qui défend la vision picturale du langage, les langues se regroupent et se recoupent autour d'images visuelles et sémantiques.

\section{Traduction et auto-traduction chez Nabokov}

Le problème du multilinguisme dans l'autobiographie nabokovienne se conjugue avec le problème de la traduction littéraire et de l'auto-traduction. Certains chercheurs (Elizabeth Klosty Beaujour, Judson Rosengrant et Jane Grayson) considèrent que la traduction et l'auto-traduction représentent, par leur diversité et leur étendue, une des parties majeures et significatives de l'œuvre de Nabokov. Selon Beaujour, dans le cas de l'auto-traduction, le texte traduit peut dépasser l'original par ses qualités artistiques et esthétiques, car son auteur le retravaille en apportant une réflexion supplémentaire au mo-

\footnotetext{
${ }^{9}$ Ibidem, p. 52-53.

${ }^{10} \mathrm{Ibidem}$, p. 24.
} 
ment de la traduction. ${ }^{11}$

Dans son ouvrage Nabokov traduit. Comparaison de la prose russe et anglaise de Nabokov [Nabokov Translated. A Comparison of Nabokov's Russian and English Prose, 1977], Jane Grayson expose la théorie de la traduction chez Nabokov et sa mise en pratique. Dans le chapitre Les traductions [The Translations], le chercheur distingue quatre axes de traduction dans l'œuvre nabokovienne: traduction en anglais, traduction en russe, traduction en anglais et en russe, les traductions du français et en français.

1) Les auto-traductions du russe en anglais débutent en 1937 avec le roman russe La méprise [Отчаяние] intitulé en anglais Despair et avec Rire dans la nuit [Laughter in the Dark], traduction anglaise de Camera obscura. A son arrivée aux États-Unis, Nabokov s'adonne à l'écriture en anglais et entame des traductions massives du russe en anglais, une fois devenu mondialement célèbre. Pendant treize ans, entre 1959 et 1977, il collabore avec ses traducteurs afin de traduire tous ses romans russes en anglais, corrige les traductions et traduit lui-même les vers se trouvant dans le corps des textes. Trente et un des cinquante récits russes ont été traduits en anglais. Il a effectué la traduction de ses trente-neuf poèmes russes pour le recueil Poèmes et problèmes [Poems and Problems, 1970].

2) Dans les années vingt et trente, Nabokov privilégie les traductions du français et de l'anglais en russe. Avec le passage à l'anglais, le russe est mis à l'écart par l'écrivain. Cependant, il continue à composer des poèmes en russe et à écrire en russe des articles pour les revues d'émigration. Les traductions en russe (Drugie berega, Lolita) sont toujours effectuées par Nabokov lui-même. Grayson considère que le degré d'exactitude de la traduction varie selon les œuvres traduites.

3) Le seul exemple qui puisse caractériser le troisième groupe est l'expérience que Nabokov a faite sur son autobiographie, dans laquelle le traducteur a entrepris deux changements de langue: le premier, de l'anglais en russe et le second, du russe

11 BEAUJOUR, 1995, p. 719. 
en anglais. Son autobiographie représente un énorme intérêt pour l'étude du rôle de la langue dans la genèse des écrits littéraires, puisqu'elle permet d'examiner les stratégies de traduction dans les deux sens.

4) Adolescent, Nabokov a traduit des passages du Cavalier sans tête de Mayne Read en français. Dans les années vingt, il a effectué plusieurs traductions du français en russe: Colas Brugnon en 1922 sous le titre Николка Персик, un sonnet de Pierre Ronsard en 1922, "L'albatros» de Charles Baudelaire en 1922, «La nuit de décembre» de Musset en 1916 et 1928 et son poème «La nuit de mai» en 1927, «Le bateau ivre» de Rimbaud en 1928. Au début de 1937 il a donné une conférence en français intitulée «Pouchkine, ou le vrai et le vraisemblable», dans le corps de laquelle il a inclus ses traductions de poèmes d'Aleksandr Puškin. Il a traduit la nouvelle russe «Musique» [«Музыка», 1932] en français, publiée dans La nouvelle revue française en 1959, l'année de son retour en Europe, et a corrigé la plupart des traductions en français.

L'originalité et la nouveauté de Drugie berega / Speak, Memory consiste dans le transfert constant, d'une langue à l'autre, du texte qui est remanié, repris, complété (russe $\circledast$ anglais $\AA$ français $\AA$ anglais $\AA$ russe $\AA$ anglais). Le bilinguisme de Nabokov n'est pas un phénomène singulier et unique dans la littérature, car cette dernière connaît bien évidemment d'autres exemples de bilinguisme d'écriture et d'auto-traduction: Julien Green, Samuel Beckett, Andreï Makine, Fernando Pessoa, Elias Canetti, Claude Esteban, etc. Dans l'article «Vladimir Nabokov et le bilinguisme culturel», Wladimir Troubetzkoy souligne que le phénomène du bilinguisme est propre à la culture russe dans la première moitié du XXème siècle, en nommant deux antécédents: le français de Puškin et l'ouverture linguistique de la culture de l'Âge d'argent. ${ }^{12}$

\footnotetext{
12 TROUBETZKOY, 1996, p. 123.

«La littérature russe du XXe siècle présente en effet un nombre élevé, anormalement élevé, d'auteurs écrivant en plusieurs langues. Les circonstances, la Révolution de 1917, l'exil y ont leur part. Mais aussi la tradition, celle dont Pouchkine, "le Français", ainsi que l'universalisme tout récent de l'Âge d'argent, sont les exemples éclatants. Chaque cas de bilinguisme d'écriture est un cas particulier, mais Elsa Triolet, Mark Aldanov, Zinaïda Chakhovskaïa, Vladimir
} 
L'arrivée de Nabokov aux États-Unis a été marquée par la publication de l'article «L'art de la traduction» [«The Art of Translation", 1941] dans The New Republic où l'écrivain défend la traduction littérale, l'art de l'imitation poussé à son extrême. Le souci linguistique de création et de traduction occupe une place importante dans l'ensemble de l'œuvre de Nabokov. L'autobiographie est une illustration en acte de la problématique de l'auto-traduction telle qu'elle se manifeste chez cet auteur. Par exemple, il existe quatre versions du texte sur la gouvernante française: une en français, deux en anglais, une en russe. La nouvelle «Mademoiselle O» parue en 1936, traduite en anglais par Hilda Ward et modifiée par Nabokov, fut publiée au magazine The Atlantic Monthly en 1943, dans Nine Stories en 1947 et Nabokov's Dozen [1958, 1959, 1960]. Elle est incorporée à la première version de l'autobiographie intitulée Conclusive Evidence [1951] qui est à son tour traduite en russe sous titre Drugie berega [1954]. Cette traduction diffère sensiblement de l'original anglais, mais, en outre, les transformations et les modifications introduites sont en partie incorporées à la dernière version anglaise Speak, Memory [1967]. Dans l'avant-propos de Speak, Memory Nabokov écrit: «Dans l'édition présente et définitive de Speak, Memory, je n'ai pas seulement apporté des modifications fondamentales au texte anglais initial, augmenté en outre d'ajouts consistants, mais j'ai également tiré profit des corrections effectuées alors que je le transposais en russe» ${ }^{13}$.

Ainsi, nous voyons que les relations extrêmement variées et complexes entre les versions successives se déploient sur trois langues différentes. Dans Bilinguisme d'écriture et auto-traduction: Julien Green, Samuel Beckett, Vladimir Nabokov, Michaël Oustinoff considère que «l'entrecroisement des textes est impressionnant, non seulement par l'ampleur de ses ramifications, mais également par les problèmes liés aux

Pozner, Vassili lanovski, David Knout, Wladimir Weidlé sont de véritables écrivains bilingues: Vladimir Nabokov n'est que le plus doué d'entre eux. D'autres se sont essayés à l'écriture en français, Dimitri Mérejkovski, Zinaïda Gippius, Marina Tsvétaiéva, Teffi, Poplavski, mais jamais de manière suivie et systématique».

${ }^{13}$ NABOKOV, 1991a, p. 15. 
changements de l'écriture ${ }^{14}$. Mais derrière cette virtuosité dans la transposition d'un texte d'une langue à l'autre se cache la difficulté extrême de cette entreprise que l'auteur souligne dans l'avant-propos de Speak, Memory:

Cette remise en forme, en anglais, d'une remise en forme en russe de ce qui avait été au départ une restitution en anglais des souvenirs russes, s'est révélée être une besogne infernale, mais je me suis quelque peu consolé en me disant que de telles métamorphoses à répétition, familières aux papillons, n'avaient encore été tentées par aucun humain..$^{15}$

L'auto-traduction de la première version anglaise Conclusive Evidence en russe sous le titre Drugie berega précède la grande période des auto-traductions réalisées dans l'autre sens (1959-1972) et s'effectue au moment où Nabokov échafaude sa théorie de la traduction littérale qui aboutit en 1964 à la traduction «littérale» d'Eugène Onéguine [Eugene Onegin]. Il formule progressivement sa doctrine de la traduction littérale qu'il défend dans des articles, des préfaces et des commentaires. L'expression "traduction littérale» apparaît dans l'article «Les problèmes de la traduction» [«Problems of Translation», 1955]. Dans la préface à Eugène Onéguine, Nabokov distingue trois types de traduction: paraphrastique, lexical et littéral. Le type paraphrastique induit des ajouts et des suppressions dans le texte traduit. Le type lexical (ou structural) rend le sens premier et l'ordre des mots. Le type littéral est le seul type de traduction qui soit reconnu par Nabokov et qui soit capable de donner la signification contextuelle des vocables de l'original ainsi que les caractéristiques associatives et syntaxiques du texte de départ. ${ }^{16}$ La traduction littérale doit rendre à la fois le sens manifeste et le sens caché du texte traduit.

Dans la traduction en russe d'Alice au pays des merveilles [Alice in Wonderland, 1865] sous le titre d'Anja au pays des merveilles [Аня в стране чудес, 1923], Nabokov utilise la méthode de transposition et remplace les images littéraires du

\footnotetext{
${ }^{14}$ OUSTINOFF, 2001, p. 266.

${ }^{15} \mathrm{NABOKOV}$, op. cit., p. 15.

${ }^{16}$ NABOKOV, 1990, vol. 1, p. vii-viii.
} 
texte original par des images équivalentes dans la littérature russe. ${ }^{17}$ Sa traduction d'Eugène Onéguine exclut transpositions et descriptions explicatives et garde la trace des images littéraires d'origine, toutes les explications étant réservées aux notes. L'expression du contenu prime sur celle de la forme dans la traduction d'Eugène Onéguine, tandis que dans la traduction des œuvres fictionnelles de Nabokov, nous assistons au phénomène inverse qui cherche à préserver l'assonance et l'allitération des textes d'origine. ${ }^{18}$ Dans la préface au recueil Poèmes et problèmes, notre auteur revient sur la question de la traduction littérale basée sur la notion de fidélité artistique:

J'ai, ces dix dernières années, prôné autant que possible la littéralité, c'est-à-dire une fidélité rigoureuse en matière de traduction poétique. C'est une façon honnête d'aborder un texte et un véritable plaisir, quand on a affaire à un chef-d'œuvre reconnu dont chaque détail doit être fidèlement rendu en anglais. Mais que faire quand il s'agit de traduire ses propres vers, écrits il y a un demi ou un quart de siècle? On doit alors lutter avec une gêne confuse ; on ne peut pas s'empêcher de se tortiller et de grimacer; on se sent un peu comme un potentat qui se jure allégeance à lui-même ou comme un prêtre consciencieux qui bénit l'eau de son propre bain. D'autre part, que dans un instant d'égarement on envisage la possibilité de refaire et d'améliorer, des années après, ses propres vers, on bat en retraite aussitôt avec l'horrible sentiment d'être coupable de falsification, pour s'accrocher comme un petit singe à une totale fidélité. Je n'ai accepté qu'un seul compromis mineur: là où c'était possible, j'ai accueilli la rime ou son ombre ; mais jamais je n'ai tordu la queue d'un vers pour l'amour de l'assonance ; et j'ai renoncé au rythme de l'original si je devais pour cela réviser le sens. ${ }^{19}$

\footnotetext{
${ }^{17}$ Cf. notre article «"My s Vremenem rassorilis' v prošlom Martobre...": lingvističeskie i kul'turologičeskie anaxronizmy v perevode V.V. Nabokova "Anja v strane čudes" (1923)», in Detskie čtenija, Saint-Pétersbourg, 2017, № 12, pp. 203-223.

18 Voir à ce sujet: Jane Grayson, Nabokov Translated. A Comparison of Nabokov's Russian and English Prose, Oxford, Oxford University Press, 1977 et Judson Rosengrant, Nabokov's Autobiography: Problems of Translation and Style, Stanford, Stanford University, 1983.
}

${ }^{19}$ NABOKOV, 1999, p. 14. 
Dans cette citation, Nabokov expose les mêmes principes pour la traduction littérale et l'auto-traduction, tout en soulignant leurs différences. Il apparaît dans ce passage comme un traducteur rationaliste qui n'hésite pas à sacrifier la forme poétique (le rythme et la rime) au sens. Par contre, le poète-traducteur fait tout son possible pour garder le mètre dans ses traductions.

Les changements de textes ont été dictés, dans le cas de notre auteur, non pas par des considérations autobiographiques, mais par un critère esthétique. Les souvenirs russes entrent en consonance avec les signifiés russes, puisqu'il existe un lien très fort entre la langue et le souvenir vécu, entre la langue et le type des souvenirs. Nous constatons que la traduction de l'écriture autobiographique est beaucoup plus libre que celle de l'écriture fictionnelle. Les réflexions sur la nature des langues et leur «transposabilité» aboutit chez Nabokov à l'invention d'une langue imaginaire dans ses romans L'exploit, Solus Rex, Brisure à senestre et Feu pâle.

\section{Relation binaire des langues russe et anglaise dans l'autobiographie de Nabokov}

La tâche de l'auto-traduction de l'autobiographie est rendue compliquée non seulement par les différences linguistiques entre l'anglais et le russe, mais aussi par le statut du vécu de l'auteur encore présent dans la mémoire de l'émigration russe et inexistant, importé d'ailleurs, d'«autres rivages» pour le public anglophone. Dans une lettre à Edmund Wilson datée du 16 janvier 1952, Nabokov évoque ce problème de la traduction de son autobiographie sur l'exemple du chapitre «Exil»:

Il [Roman Grynberg - S. G.] a traduit le chapitre «Exil» de Concl[usive] Evi[dence] en russe, mais je constate maintenant avec horreur et stupéfaction que ce fut peine perdue - le russe sonne terriblement agressif, et ne peut être publié tant que les gens auxquels je fais allusion, à travers mon voi- 
le américain (que la traduction arrache, bien sûr), sont encore en vie. ${ }^{20}$

L'introduction des signifiés russes dans le texte autobiographique en anglais n'engage aucun travail de la mémoire pour le lecteur anglophone. Cette absence de souvenirs crée le «voile américain", ce qui permet à l'écrivain d'avoir plus de liberté dans son œuvre autobiographique. La traduction en russe fait disparaître cette faculté, ce qui rend impossible la traduction littérale. L'auto-traduction exige que l'écrivain soit plus précis: «... j'ai revu de nombreux passages et je me suis efforcé de remédier aux imperfections du texte originel, dues aux défaillances de ma mémoire: blancs, zones floues, passages imprécis» ${ }^{21}$.

Drugie berega est-il une traduction ou un original de l'autobiographie de Nabokov? Cette distinction s'avère-t-elle pertinente? Par ce problème, nous reposons, dans des termes nouveaux, la question du modèle. Rappelons d'ailleurs que son dernier roman inachevé s'intitulait justement L'original de Laura (The Original of Laura). Dans la préface de Drugie berega, la première version anglaise Conclusive Evidence est présentée comme base et original de la version russe: «Основой и отчасти подлинником этой книги послужило ее американское издание, Conclusive Evidence»"2 («Ce livre a pour point de départ et en partie pour original l'édition américaine intitulée Conclusive Evidence»²3). Dans Drugie berega, Nabokov emploie le vocable «оригинал» tantôt pour désigner la première version anglaise de l'autobiographie ( $\mathrm{B}$ оригинале этой книги...» ${ }^{24}$ / «Dans l'original de ce livre...»), tantôt pour désigner la vie en évoquant l'opposition «vie / écriture». L'original est la vie, exprimée en images et souvenirs, traduite en caractères visibles et transposée en différentes langues:

\footnotetext{
${ }^{20}$ NABOKOV; WILSON, 1988, p. 298.

${ }^{21}$ NABOKOV, 1991a, p. 15.

${ }^{22}$ NABOKOV, 1978, p. 7.

${ }^{23}$ NABOKOV, 1991b, p. 393.

${ }^{24} \mathrm{NABOKOV}, 1978$, p. 35.
} 
Всякий раз, что удавалось посетить Прагу, я испытывал в первую секунду встречи ту боль, ту растерянность, тот провал, когда приходится сделать усилие, чтобы нагнать время, ушедшее за разлуку вперед, и восстановить любимые черты по нестареющему в сердце образцу. [...] Около ее кушетки, ночью служившей постелью, ящик, поставленный вверх дном и покрытый зеленой материей, заменял столик, и на нем стояли маленькие мутные фотографии в разваливающихся рамках. Впрочем, она едва ли нуждалась в них, ибо оригинал жизни не был утерян. ${ }^{25}$

Chaque fois que j'avais l'occasion de visiter Prague, au premier moment de la rencontre j'éprouvais cette douleur, ce désarroi, ce défaut de mémoire quand il fallait faire un effort afin de rattraper le temps qui avait avancé pendant la séparation et afin de restaurer les traits aimés d'après le modèle intact qui se trouve dans le cœur. [...] A côté de sa couchette qui servait de lit la nuit, un coffre renversé et drapé de tissu vert remplaçait une table basse sur laquelle étaient posées de petites photographies ternes dans des cadres en voie de désagrégation. D'ailleurs, elle avait à peine besoin d'elles, car l'original de la vie n'était pas perdu.

Nabokov évoque ici la question du modèle qu'il garde dans sa mémoire («по нестареющему в сердце образцу»). L'expression «нагнать время» précise que l'autobiographe interpose deux images de sa mère: celle sauvegardée par sa mémoire et celle de la vie. Ce passage montre que l'autobiographe tient cette faculté de sa mère qui a su préserver dans sa mémoire l'original de la vie («оригинал жизни»), original qu'elle se remémore dans le présent.

Les différentes autobiographies sont donc des versions d'un original qui se trouve dans une zone prélangagière qui à la fois n'est exprimée en aucune langue et est exprimée en toutes les langues. Les images tracées par la vie de l'écrivain dans sa mémoire sont traduites en différentes langues. L'expérience visuelle est l'original de l'autobiographie de Nabokov, ce qui expliquerait l'importance de la coordination des thèmes, des motifs dans Drugie berega / Speak, Memory. Dans l'envoi de

${ }^{25}$ Ibidem, p. 41. 
Littératures II, l'écrivain déclare que l'art est universel et que c'est la langue qui le fractionne en versions nationales: «La langue est la seule chose qui fragmente un art universel en arts nationaux. [...] ... la littérature n'appartient pas au domaine des idées générales mais à celui des mots et des images spécifiques ${ }^{26}$.

Remarquons que Nabokov introduit le problème de l'auto-traduction dans la préface de Drugie berega et l'avant-propos de Speak, Memory. Tout le long du texte russe, il fait des digressions qui résument les explications données dans la version anglaise. Dans la préface russe, l'autobiographe a inclus, entre autres choses, les moments cruciaux de sa biographie artistique tels que la nécessité vitale de changer d'idiome de création. ${ }^{27}$

\section{Drugie berega et Lolita: problème du pas- sage de l'anglais au russe}

L'autobiographie de Nabokov et son roman le plus célèbre Lolita sont étroitement liés par la simultanéité de la création et par le processus de l'auto-traduction d'anglais en russe. Ces deux livres sont la seule utilisation «active» du russe dans la prose après l'abandon de cette langue en 1939 (pourtant le poète n'a jamais abandonné l'écriture des vers en russe). Alfred Appel, dans son introduction au livre The Annotated Lolita, souligne les rapports qui se nouent entre Speak, Memory, ouvrage le plus autobiographique, et Lolita, ouvrage le moins autobiographique et le plus romanesque de Nabokov. ${ }^{28}$ Selon

\footnotetext{
${ }^{26}$ NABOKOV, 1985b, p. 426.

${ }^{27}$ NABOKOV, 1978, p. 7-8.

${ }^{28}$ APPEL, 1991, p. xxiii.

"As a book about the spell exerted by the past, "Lolita" is Nabokov's own parodic answer to his previous book, the first edition of "Speak, Memory". Mnemosyne is now seen as a black muse, nostalgia as a grotesque cul-de-sac. "Lolita" is the last book one would offer as "autobiographical", but even in its totally created form it connects with the deepest reaches of
} 
ce chercheur, le thème autobiographique est transformé dans l'écriture fictionnelle de Lolita: «An autobiographical theme submitted to the imagination thus takes on a new life: frozen in art, halted in space, now timeless, it can be lived with». ${ }^{29}$

Drugie berega est le livre le plus fidèle à la "réalité», au souvenir. Lolita est son livre de fiction le plus hardi et qui rompt avec la perception conventionnelle du monde réel. Nabokov le dit dans Intransigeances: "Autres rivages est un livre strictement autobiographique. Il n'y a rien d'autobiographique dans Lolita, "30. Dans l'avant-propos de Speak, Memory, il évoque le lien d'écriture qui existe entre son œuvre autobiographique et ses œuvres fictionnelles:

Durant l'été 1953, dans un ranch des environs de Portal, Arizona, dans une maison louée à Ashland, Oregon, et dans divers motels de l'Ouest et du Mid-west, j'ai trouvé le moyen, tout en chassant les papillons et en écrivant Lolita et Pnine, de traduire en russe Speak, Memory avec le concours de ma femme. ${ }^{31}$

L'autobiographie en russe paraît en 1954 et Lolita en anglais est publié en 1955. Dans les années soixante, Nabokov entreprend la traduction de ces deux œuvres et l'autobiographie en anglais et Lolita en russe sont édités la même année, en 1967. Lolita et l'autobiographie forment donc une opposition et non seulement par la concomitance de l'écriture. Dans ces deux œuvres, exactement les mêmes thèmes sont traités, mais d'une manière différente, oppositionnelle: rapports entre passé, présent et futur, entre temps et espace, entre réalité et rêve, vérité et fiction, entre mémoire, souvenirs et imagination ; structure de motifs; thème du clair-obscur (ombre, reflet, miroir, soleil, lune); techniques propres au théâtre et au conte; figure du destin, de la Muse et de Mnémosyne; personnage du lecteur et emploi du français dans le texte. De plus, notons que le grand romancier russo-américain a publié seulement deux

\footnotetext{
Nabokov's soul».

${ }^{29}$ Ibidem, p. xxiii.

${ }^{30}$ NABOKOV, 1985, p. 80.

${ }^{31}$ NABOKOV, 1991a, p. 15.
} 
romans en russe sous son vrai nom, Drugie berega et Lolita, les autres romans russes sont parus sous son pseudonyme Sirin. Disons à ce propos quelques mots sur le nom de plume sous lequel Nabokov était connu de l'émigration russe. Le nom de plume Sirin, pour reprendre la définition de l'écrivain lui-même, «oiseau multicolore, avec un buste et un visage de femme», a une connotation riche et complexe. Le champ sémantique de ce nom s'étend, entre autres, à la nymphe et à la nymphette. Ce dernier vocable a été inventé par Nabokov, le concept correspondant se traduit par la création de multiples personnages féminins dont les plus célèbres sont Lolita, Ada et Laura, personnages par lesquels la figure de Sirin apparaît discrètement dans les romans américains. De plus, dans la version russe d'«À propos d'un livre intitulé Lolita (Postface à l'édition américaine de 1958)" ["О книге, озаглавленной Лолита (Послесловие к американскому изданию 1958 года)», 1958], en parlant de la genèse du roman issu du texte russe inachevé L'enchanteur (1939), Nabokov fait transparaître son pseudonyme au moyen d'une métaphore «les griffes et les ailes d'un roman» et du vocable «nymphette» dont le concept reste inchangé («la nymphette est restée la même»):

Несмотря на смесь немецкой и ирландской крови вместо одной французской, нимфетка осталась той же, и тема женитьбы на матери - в основе своей - тоже не изменилась; но в других смыслах вещь приняла совершенно новый вид; у нее втайне выросли когти и крылья романа. ${ }^{32}$

Malgré le mélange d'un sang irlandais et allemand au lieu d'un sang uniquement français, la nymphette est restée la même, et le thème du mariage avec sa mère n'a pas, au fond, non plus changé ; mais dans d'autres sens, la chose a acquis un aspect tout à fait nouveau ; les griffes et les ailes d'un roman y ont poussé secrètement.

Dans son article «Nabokov. La première Lolita», Vladimir Weidlé, critique célèbre de l'émigration russe, caractérise ain-

\footnotetext{
32 NABOKOV, 2003a, t. 2, p. 378. L'article fut écrit en anglais le 12 novembre 1956 pour The Anchor Review (Doubleday, New York), en 1958 il fut intégré à l'édition américaine de Lolita chez G. P. Putnam's Sons, New York.
} 
si le roman le plus connu de notre auteur:

Книга эта, как о ней «в конечном счете» ни суди, останется навек самым зрелым произведением совершенно исключительно одаренного, редкостно блестящего русского писателя. [...] Я дажеглубокоубежден, что русская «Лолита» гораздо более крупное явление русской литературы, чем английская английской (или американской). ${ }^{33}$

Ce livre, peu importe comment on le juge «à la fin», restera à jamais l'œuvre la plus mûre d'un écrivain russe singulièrement doué et tout particulièrement brillant. [...] Je suis même profondément convaincu que la Lolita russe est un événement beaucoup plus important pour la littérature russe que celle écrite en anglais pour la littérature anglaise (ou américaine).

Justement, Nabokov termine "À propos d'un livre intitulé Lolita» par le constat que son premier idiome littéraire, la langue russe, dominera toujours l'idiome d'adoption, la langue anglaise. Il souligne, une fois de plus, son attachement particulier au fonctionnement de la langue en tant que pilier structurant d'une œuvre littéraire, en comparant ses outils langagiers en russe aux accessoires de l'illusionniste. En recourant à la métaphorique de l'illusionnisme, l'artiste montre que son style est fondé sur l'appropriation et le dépassement du modèle russe, déformé («каверзного зеркала» / «le miroir trompeur») et dissimulé («черно-бархатного задника» / «la toile de fond au velours noir») dans ses écrits. ${ }^{34}$ La référence au métier de l'illusionniste soulève aussi la question de l'artificialité manifeste, ostensible de l'écriture nabokovienne.

$\mathrm{Au}$ début du «Post-scriptum à l'édition russe» [«Постскриптум к русскому изданию», 1967] de Lolita, Nabokov relativise la déclaration qui figure à la fin d'« $\dot{A}$ propos d'un livre intitulé Lolita» et proclame la supériorité de son style russe sur son style anglais. Cette déclaration est relativisée suite à l'auto-traduction de Lolita d'anglais en russe effectuée

${ }^{33}$ VEJDLE, 2001, p. 867-868.

${ }^{34}$ NABOKOV, op. cit., p. 385. 
entre 1963 et 1965 et suite à la non-correspondance des attentes de l'écrivain présentées sous l'image du printemps et du produit fini comparé à un tableau automnal dans le passage ci-dessous:

История этого перевода - история разочарования. Увы, тот «дивный русский язык», который, сдавалось мне, все ждет меня где-то, цветет, как верная весна за наглухо запертыми воротами, от которых столько лет хранился у меня ключ, оказался несуществующим, и за воротами нет ничего, кроме обугленных пней и осенней безнадежной дали, а ключ в руке скорее похож на отмычку. ${ }^{35}$

L'histoire de cette traduction est l'histoire d'une déception. Hélas, cette «langue russe divine» qui, me semblait-il, m'attendait encore quelque part, fleurissait comme un printemps fidèle derrière des portes condamnées dont j'avais gardé la clé pendant tant d'années, s'est avérée inexistante, et, derrière les portes, il n'y a que des souches carbonisées et un horizon lointain automnal désespéré, et la clé dans la main ressemble plutôt à un passe-partout.

La traduction de Lolita d'anglais en russe met en évidence la question de la «traduisibilité» des deux langues, ce qui est reflété également dans l'auto-traduction de son autobiographie. Nabokov illustre la différence de ces deux langues au niveau historique en développant un réseau de comparaisons végétales («между зеленым русским литературным языком и зрелым, как лопающаяся по швам смоква, языком английским» / «entre la verte langue littéraire russe et la langue anglaise mûre comme une figue craquant aux coutures») et humaines («между гениальным, но еще недостаточно образованным, а иногда довольно безвкусным юношей, и маститым гением, соединяющим в себе запасы пестрого знания с полной свободой духа» / «entre un jeune homme de génie, mais pas encore suffisamment instruit et parfois assez dépourvu de goût et un génie vénérable conjuguant en lui un grand fonds d'érudition bigarrée avec une entière liberté d'esprit») et en mettant au jour le principe primordial inhérent

${ }^{35}$ NABOKOV, 2003b, p. 386. 
à la langue et à la culture anglophones, celui de la liberté de l'esprit. ${ }^{36}$

A la fin du «Post-scriptum à l'édition russe» de Lolita, Nabokov désigne un des objectifs de cette auto-traduction: traduire avec exactitude une de ses meilleures œuvres de la langue d'adoption dans sa langue maternelle et sa première langue de l'écriture littéraire: «Издавая «Лолиту» по-русски, я преследую очень простую цель: хочу, чтобы моя лучшая английская книга - или, скажем еще скромнее, одна из лучших моих английских книг - была правильно переведена на мой родной язык. Это - прихоть библиофила, не более» ${ }^{37}$ («En publiant Lolita en russe je poursuis un but très simple: je veux que mon meilleur livre anglais ou bien, disons encore plus modestement, l'un de mes meilleurs livres anglais, soit traduit correctement en ma langue maternelle. Ce n'est qu'une lubie de bibliophile»).

La figure de l'auteur est décomposée en trois entités dans ce texte: celle de l'écrivain, celle du traducteur et celle du lecteur. Comme traducteur, l'artiste tient à souligner sa volonté de ne pas introduire des blancs et des ajouts dans la traduction russe de Lolita. Il est à remarquer que dans le commentaire de Lolita, A. M. Ljuksemburg doute de la fidélité de la traduction. D'après lui, Nabokov prend des libertés avec l'original par endroits et la deuxième moitié du texte est parsemée de modifications soigneusement dissimulées qui accentuent le caractère conventionnel du récit. ${ }^{38}$

Dans le «Post-scriptum à l'édition russe», l'écrivain engendre ses lecteurs-marionnettes qu'il manipule et qui apparaissent dans la narration sous l'aspect de ses doubles obéissant passivement aux ordres suprêmes de l'artiste. Nabokov distingue ici deux types de lecteurs: un lecteur qui est une exacte réplique de lui-même et qui est utilisé à des fins artistiques spécifiques, et un lecteur normal, un lecteur «autre» que l'écrivain ne

\footnotetext{
${ }^{36}$ Ibidem, p. 386-387.

37 Ibidem, p. 389.

${ }^{38}$ Ljuksemburg, 2003, p. 601.
} 
peut pas prévoir et contrôler. A l'aide du cristal magique, image empruntée à Aleksandr Puškin, encrier-symbole de l'art et de l'écriture, l'artiste essaie de se souvenir de son lecteur du passé et de déterminer son lecteur futur en se transportant dans son imagination à Moscou. D’abord, sont énumérés ses lecteurs du passé, lecteurs qu'il a connus, décrits sur un mode assez ironique. Tout à la fin du post-scriptum, le romancier rejoue, dans son imagination, le retour en Russie: l'accueil de l'écrivain à son arrivée par avion (cette scène sera rejouée plus tard dans Regarde, regarde les arlequins .). Le narrateur-écrivain est déçu, car il ne rencontre pas son lecteur de jeune génération. Ce procédé de trompe-l'œil a pour but de montrer que le lecteur des générations futures est un mirage, une donnée inaccessible à l'écrivain.

Notons, entre autres, que le roman Pnine [1957] fait aussi une large part à la question des langues (le thème des langues reviendra dans les romans Feu pâle [1962] et Ada ou l'ardeur [1969], mais il aura perdu de sa gravité et sera le prétexte à d'éblouissants jeux multilingues). Pour le protagoniste Timofeï Pnine, l'anglais est la langue du déracinement, à l'image des États-Unis où il va de meublé en chambre d'hôte, sans s'attacher à aucun lieu. Le roman pose la question du sens de la transition entre la langue maternelle et la langue de l'exil. Nabokov abandonne son héros à mi-chemin entre deux langues et deux mondes, traduisant probablement ses propres incertitudes de romancier converti à l'écriture en anglais, soulevant le dilemme de l'écrivain bilingue et le traumatisme du passage d'une langue à une autre, car le choix de l'écriture en anglais, bien que délibéré, représentait pour lui une tragédie personnelle. Il le montre d'ailleurs dans Intransigeances. «le passage, sans retour, de la prose russe à la prose anglaise fut douloureux à l'extrême - c'était un peu comme réapprendre à manier des objets après avoir perdu sept ou huit doigts dans une explosion» ${ }^{39}$.

${ }^{39}$ NABOKOV, 1985a, p. 66. 
Dans le cas de Vladimir Nabokov il s'agit d'un "croisement», d'une «hybridation» des cultures conduite par le biais d'une «hybridation» des langues. Le va-et-vient translinguistique traverse toutes les versions de l'autobiographie, ce que Georges Nivat montre dans son article "Speak, Memory» sur l'exemple de l'épisode du père Crépin: «The two versions of the passage about "père Crépin" are in fact complementary; here, as in many other cases, the English version and the Russian one form a literary "Janus bifrons", a two-faced text, a poetic interface $»^{40}$. La représentation du texte autobiographique comme «Janus bifrons» permet de discerner la théorie linguistique de Nabokov qui consiste à rechercher une langue universelle, celle de la création artistique, le sens étant transféré d'une langue à l'autre par le biais d'images visuelles.

De la même manière qu'Aleksandr Blok, selon Osip Mandel'štam, comprenait le renouveau de la langue et de la littérature russes comme croisement de différentes formes littéraires, croisement qui crée un style et l'améliore ${ }^{41}$ de même Nabokov réalise ce principe sur le plan multilingue, en essayant de créer des ponts sémantiques entre trois langues qui permettront d'intégrer ces dernières dans un unique système linguistique.

Comme chaque langue représente un monde particulier, le traducteur doit rendre ce monde particulier dans un autre système linguistique. Les recherches nabokoviennes dans le domaine de la traduction littéraire peuvent être mises en perspective avec les conceptions de Walter Benjamin telles qu'il les expose dans son article «La tâche du traducteur» dans lequel le théoricien souligne la chose suivante:

Ainsi la finalité de la traduction consiste, en fin de compte, à exprimer le rapport le plus intime entre les langues. Il lui est impossible de révéler, de créer ce rapport caché lui-même ; mais elle peut le représenter en le réalisant en germe ou intensivement. Et cette représentation d'un signifié par l'essai, par le germe de sa création, est un mode de représen-

\footnotetext{
40 Nivat, 1995, p. 681.

41 Mandel'štam, 1990, t. 2, p. 190.
} 
tation tout à fait original, qui n'a guère d'équivalent dans le domaine de la vie non langagière. ${ }^{42}$

La référence à Walter Benjamin permet de mieux comprendre les raisons du multilinguisme nabokovien. Pour notre auteur, la traduction doit signifier et faire apparaître à la conscience du lecteur ce qui ne relève d'aucun idiome: l'«entre-langues», le rapport entre les langues, l'intervalle qui présuppose à la fois leur essentielle unité et leur irréparable altérité.

\section{Références bibliographiques}

APPEL, Alfred. «Introduction», in Vladimir Nabokov, The Annotated Lolita. London: Penguin Books, 1991.

BEAUJOUR, Elizabeth Klosty. "Translation and Self-translation", in The Garland Companion to Vladimir Nabokov, Vladimir E. Alexandrov (éd.). New York: Taylor and Francis Group, 1995.

BENJAMIN, Walter. "La tâche du traducteur», Traduit de l'allemand par Maurice de Gandillac, revu par Rainer Rochlitz, $\mathbb{E u -}$ vres I. Paris: Gallimard; Folio, 2000.

GARZIANO, Svetlana. La poétique autobiographique de Vladimir Nabokov dans le contexte de la culture russe et occidenta$l e$, préf. de Jean-Claude Lanne, Specimina Slavica Lugdunensia IV. Lyon: CESAL, 2012.

««My s Vremenem rassorilis' v prošlom Martobre...»: lingvističeskie i kul'turologičeskie anaxronizmy $\mathrm{v}$ perevode V.V. Nabokova «Anja v strane čudes» (1923)», in Detskie čtenija. Saint-Pétersbourg: 2017, № 12, pp. 203-223.

GRAYSON, Jane Nabokov Translated. A Comparison of Nabokov's Russian and English Prose. Oxford: Oxford University Press, 1977.

LJUKSEMBURG, A. M. «Kommentarii. Lolita», in V. V. Nabokov, Amerikanskij period. Sobranie sočinenij $v 5$ tomax, 2003, t. 2.

42 BENJAMIN, 2000, p. 248. 
MANDEL'ŠTAM, Osip. «A. Blok», Sočinenija v 2 tomax. Moskva: Xudožestvennaja literatura, 1990, t.2.

NABOKOV, Vladimir; WILSON, Edmund. Correspondance, 19401971. Traduit de l'anglais par Christine Raguet-Bouvart. Paris: Rivages, 1988.

NABOKOV, Vladimir. «À propos d'Autres rivages». Traduit de l'anglais par Maurice Couturier, in N. R. F., septembre 1999, $\mathrm{n}^{\circ}$ 551.

Autres rivages. Traduit du russe par Laure Troubetzkoy. Paris: Gallimard, 1991a. Drugie berega. Ann Arbor: Ardis, 1978. «Foreword», in: Aleksandr Pushkin, Eugene Onegin. Introduction and Translation. Traduit du russe en anglais par Vladimir Nabokov. Princeton: Princeton University Press, Bollingen Series LXXII, 1990, vol. 1.

Intransigeances. Traduit de l'anglais par Vladimir Sikorsky. Paris: Julliard, 1985a.

. «Introduction», Poèmes et problèmes. Traduit de l'anglais par Hélène Henry. Paris: Gallimard, 1999.

Kamera obscura, Russkij period. Sobranie sočinenij $v$ 5 tomax. Sankt-Peterburg: Simpozium, 2001, t. 3.

Littératures II. Gogol, Tourguéniev, Dostoïevski, Tolstoï, Tchekhov, Gorki. Traduit de l'anglais par Marie-Odile Fortier-Masek. Paris: Fayard, 1985b.

"O knige, ozaglavlennoj "Lolita" (Posleslovie k amerikanskomu izdaniju 1958 goda)» [«A propos d'un livre intitulé Lolita (Postface à l'édition américaine de 1958)»], Amerikanskij period. Sobranie sočinenij $v 5$ tomax, 2003a, t. 2.

"Postskriptum k russkomu izdaniju», Lolita, Amerikanskij period. Sobranie sočinenij v 5 tomax, 2003b, t. 2. «Préface à l'édition russe», Autres rivages, Paris, Gallimard, 1991b.

NIVAT, Georges. «Speak, Memory», in The Garland Companion to Vladimir Nabokov, Vladimir Alexandrov (éd.), New York, Taylor and Francis Group, 1995. 
OUSTINOFF, Michaël. Bilinguisme d'écriture et auto-traduction: Julien Green, Samuel Beckett, Vladimir Nabokov, Paris, L'Harmattan, 2001.

ROSENGRANT, Judson. Nabokov's Autobiography: Problems of Translation and Style. Stanford: Stanford University, 1983. TROUBETZKOY, Wladimir. «Vladimir Nabokov et le bilinguisme culturel», in Études russes, Mélanges offerts au professeur Louis Allain, Irina et Gilles Fougeron (éds). Lille: Presses Universitaires du Septentrion, 1996.

VEJDLE, Vladimir. «Nabokov. Pervaja Lolita», in V. V. Nabokov, pro et contra 2. Sankt-Peterburg: Izdatel'stvo Russkogo Xristianskogo gumanitarnogo instituta, 2001. 